\title{
Apurinic/apyrimidinic endonuclease 1/reduction-oxidation effector factor-1 (APE1) regulates the expression of NLR family pyrin domain containing 3 (NLRP3) inflammasome through modulating transcription factor NF-KB and promoting the secretion of inflammatory mediators in macrophages
}

\author{
Zheng Tang ${ }^{1,2,3}$, Ying Wang ${ }^{2}$, Yue Wan ${ }^{2}$, Yue $\mathrm{Xie}^{2}$, Shujie $\mathrm{Li}^{2}$, Dan Tao ${ }^{2}$, Can Wang ${ }^{2}$, Yong-Zhong Wu ${ }^{2}$, \\ Jiang-Dong Sui $^{2} \wedge$ \\ ${ }^{1}$ College of Bioengineering, Chongqing University, Chongqing, China; ${ }^{2}$ Radiation Oncology Center, Chongqing University Cancer Hospital, \\ Chongqing, China; ${ }^{3}$ Chongqing Key Laboratory of Translational Research for Cancer Metastasis and Individualized Treatment, Chongqing \\ University Cancer Hospital, Chongqing, China \\ Contributions: (I) Conception and design: JD Sui, Z Tang; (II) Administrative support: YZ Wu; (III) Provision of study materials or patients: Y Wan, \\ Y Xie; (IV) Collection and assembly of data: S Li, D Tao; (V) Data analysis and interpretation: C Wang; (VI) Manuscript writing: All authors; (VII) \\ Final approval of manuscript: All authors. \\ Correspondence to: Jiang-Dong Sui; Yong-Zhong Wu. Chongqing University Cancer Hospital, No. 181 Hanyu Road, Shapingba District, Chongqing \\ 400030, China. Email: jiangdong.sui@hotmail.com; cqmdwyz@163.com.
}

\begin{abstract}
Background: Inflammatory mediators play an important role in the occurrence, development, and metastasis of tumors. The aim of the present study was to elucidate the effect of apurinic/apyrimidinic endonuclease 1/reduction-oxidation effector factor-1 (APE1) on inflammatory mediator secretion, which is dependent on the APE1-mediated NLR family pyrin domain containing 3 (NLRP3) regulatory mechanism.

Methods: The human myeloid leukemia mononuclear cell line (THP-1) cells were cultured and polarized to M2 subset macrophages. Enzyme-linked immunosorbent assay was used for determining tumor necrosis factor- $\alpha$ (TNF- $\alpha$ ), interleukin (IL)-1 $\beta$, IL-18, IL-10, and IL-33 levels. Reverse transcription-polymerase chain reaction and western blot were used for evaluating TNF- $\alpha$, NLR family pyrin domain containing 1 (NLRP1), NLRP3, caspase-1, and apoptosis-associated speck-like protein containing a card expression. Plasmid silencing APE1 gene (APE1 $1^{\text {shRA }}$ ) was synthesized and packaged into lentiviral. For activating inflammasomes, M2-type THP-1 cells were transfected with lentiviral vector APE1 ${ }^{\text {shRNA }}$ incubated with lipopolysaccharide (LPS) (100 ng/mL)/APE1 inhibitor (E3330, $20 \mu \mathrm{M})$ and ATP. Electrophoretic mobility shift assay and dual-luciferase reporter assay were used for determining the interaction between NLRP3 and nuclear factor- $\kappa \mathrm{B}(\mathrm{NF}-\kappa \mathrm{B})$ molecule.

Results: APE1 significantly induced LPS-induced pro-inflammatory cytokine production, including TNF- $\alpha$, IL-1 $\beta$, and IL18, compared with THP-1 cells without APE1 treatment $(\mathrm{P}<0.05)$. APE1 promoted LPS-induced NLRP3 inflammasome activation by modulating the gene transcription of NLRP3-associated molecules. APE1 enhanced LPS-induced NLRP3 inflammasome activation by regulating NLRP3 and caspase-1 protein expression. APE1 improved NLRP3 activity by modulating the interaction between NLRP3 and NF- $\kappa \mathrm{B}$, and the modulation of NF- $\mathrm{BB}$. APE1 promoted LPS-induced NLRP3 inflammasome activation through an NF-кB-dependent pathway.
\end{abstract}

Conclusions: APE 1 regulates the expression of NLRP3 by modulating transcription factor NF- $\kappa \mathrm{B}$ and further promoting the secretion of inflammatory mediators IL-1 $\beta$ and IL-18 in macrophages. The

\footnotetext{
^ ORCID: Zheng Tang, 0000-0001-5945-4048; Jiang-dong Sui, 0000-0001-5171-1978.
} 
findings of the present study provide theoretical and experimental bases for the design of tumor-associated macrophage (TAM)-targeted therapy, with APE1 as the target molecule.

Keywords: Apurinic/apyrimidinic endonuclease 1/reduction-oxidation effector factor-1 (APE1); NLR family pyrin domain containing 3 inflammasome (NLRP3 inflammasome); macrophages; nuclear factor- $\kappa \mathrm{B}(\mathrm{NF}-\kappa \mathrm{B})$; inflammatory mediators

Submitted Nov 11, 2020. Accepted for publication Dec 26, 2020.

doi: 10.21037/atm-20-7752

View this article at: http://dx.doi.org/10.21037/atm-20-7752

\section{Introduction}

Inflammatory mediators are important components of the innate immune system, and play an important role in the occurrence, development, and metastasis of tumors; immune surveillance; and sensitivity of tumors to chemoradiotherapy $(1,2)$. Tumor-associated macrophages (TAMs) are the main inflammatory-mediated secretory cells in tumor stroma, which mainly secrete interleukin (IL)$1 \beta$, IL-18, matrix metalloprotein, and vascular endothelial growth factor (3). In some cases, TAMs even can constitute $50-80 \%$ of the solid tumor cell population (4). TAMs can promote tumor growth, invasion, metastasis, angiogenesis, matrix degradation, remodeling, and inhibit the acquired immunity (5). Therefore, TAMs play critical roles in the inflammatory-mediated occurrence and progression of tumors.

It has been found that NLR family pyrin domain containing 1 (NLRP1) and 3 (NLRP3) are widely expressed in tissues and cells of the whole body, and a high expression in the monocyte macrophage system (6). When tumor cells undergo changes in microenvironment, such as hypoxia and radiotherapy/chemotherapy, oxidative stress usually occurs in the TAMs, with the production of endogenous reactive oxygen species (ROS) $(7,8)$. The produced ROS in TAMs could promote the binding of NLRP1/NLRP3 proteins with apoptosis-associated speck-like protein containing a card (ASC) and/or caspase-1 to form a high molecular weight protein complex inflammasome $(9,10)$. Subsequently, caspase- 1 is activated and involved in the activation and secretion of IL-1 $\beta$ and IL- 18 . However, the redox mechanism of NLRP1 and NLRP3 in TAMs is still unclear.

Apurinic/apyrimidinic endonuclease 1/reductionoxidation effector factor-1 (APE1) is a unique bi-functional oxidative stress response protein that can activate activating protein-1 (AP-1), nuclear factor- $\kappa \mathrm{B}(\mathrm{NF}-\kappa \mathrm{B})$, and other transcription factors through redox to regulate cell proliferation, differentiation, and inflammatory response (11-13). A bioinformatics analysis also showed that both NLRP1 and NLRP3 gene promoters contain potential $\mathrm{AP}-1$ and NF- $\kappa \mathrm{B}$ binding sites (14). Therefore, we speculate that APE1 may regulate the expression of NLRP1 and NLRP3 in TAMs by activating transcription factors.

The aim of the present study was to elucidate the effect of APE1 on the secretion of inflammatory mediators by TAMs, which is dependent on the APE1-mediated NLRP1 and NLRP3 regulatory mechanism. The study provides theoretical and experimental bases for the design of TAMtargeted therapy, with APE1 as the target molecule.

We present the following article in accordance with the MDAR reporting checklist (available at http://dx.doi. org/10.21037/atm-20-7752).

\section{Methods}

\section{Cell culture and macrophages polarization}

The human myeloid leukemia mononuclear cell line, THP-1, was purchased from American Type Cell Culture Cell Bank (Category No. TIB-202, Manassas, VA, USA). THP-1 cells were cultured with the RPMI-1640 medium (Gibco, Grand Island, NY, USA) containing $100 \mu \mathrm{g} / \mathrm{mL}$ streptomycin (Beyotime Biotechnology, Shanghai, China) and $100 \mathrm{U} / \mathrm{mL}$ penicillin (Beyotime Biotechnology, China), and supplemented with $10 \%$ fetal bovine serum (Gibco, USA) at $37{ }^{\circ} \mathrm{C}$ with $5 \% \mathrm{CO}_{2}$. M2 macrophage polarization was carried out, as described in a previous study (15), with modifications. Briefly, the THP-1 cells were adjusted to a density of $1 \times 10^{6}$ cells $/ \mathrm{mL}$ medium and treated with $200 \mathrm{ng} / \mathrm{mL}$ phorbol-12-Myristate-13-Acetate (PMA) for $6 \mathrm{~h}$. THP-1 cells were polarized into M2 subset macrophages by incubating with IL-4 $(20 \mathrm{ng} / \mathrm{mL})$ and IL$13(20 \mathrm{ng} / \mathrm{mL})$ for $18 \mathrm{~h}$. M2 subset macrophages were 
Table 1 Specific primers for reverse transcription-polymerase chain reaction assay used in present study

\begin{tabular}{|c|c|}
\hline Genes & Gene sequences $\left(5^{\prime}-3^{\prime}\right)$ \\
\hline \multicolumn{2}{|l|}{$T N F-\alpha$} \\
\hline Forward & CCTGGTATGAGCCCATCTATCTG \\
\hline Reverse & AGAGGTTGAGGGTGTCTGAAGG \\
\hline \multicolumn{2}{|l|}{ NLRP1 } \\
\hline Forward & CCCTGGTCAAGAGAAGCTGG \\
\hline Reverse & AGCCCCCTGCAGTATGACTA \\
\hline \multicolumn{2}{|l|}{$N L R P 3$} \\
\hline Forward & AGGAGGACTTCGTGCAAAGG \\
\hline Reverse & GTGACTCCACCCGATGACAG \\
\hline \multicolumn{2}{|l|}{ Caspase-1 } \\
\hline Forward & CCTGCCGTGGTGATAATGTT \\
\hline Reverse & GCATCTGCGCTCTACCATCT \\
\hline \multicolumn{2}{|l|}{ ASC } \\
\hline Forward & TGGATGCTCTGTACGGGAAG \\
\hline Reverse & CCAGGCTGGTGTGAAACTGAA \\
\hline \multicolumn{2}{|l|}{$\beta$-actin } \\
\hline Forward & ACACCCAGCCATGTACGT \\
\hline Reverse & ATGGGCACAGTGTGGGTGA \\
\hline \multicolumn{2}{|c|}{$\begin{array}{l}\text { ASC, apoptosis-associated speck-like protein containing a } \\
\text { card; NLRP1, NLR family pyrin domain containing } 1 \text {; NLRP3, } \\
\text { NLR family pyrin domain containing } 3 \text {; TNF- } \alpha \text {, tumor necrosis } \\
\text { factor- } \alpha \text {. }\end{array}$} \\
\hline
\end{tabular}

sorted and by flow cytometry with the CD206 biomarker.

\section{Enzyme-linked immunosorbent assay (ELISA) measurement for cytokines}

The supernatants of culture medium were collected from polarized M2-type THP-1 cells (M2 macrophages) by centrifuging for $15 \mathrm{~min}$ at $450 \times \mathrm{g}$. In the present study, cytokines, including tumor necrosis factor- $\alpha$ (TNF- $\alpha$ ), IL1 $\beta$, IL-18, IL-10, and IL-33, were analyzed with a human TNF- $\alpha$ ELISA kit (Category No. PT518), human IL-1 $\beta$ ELISA kit (Category No. PI305), human IL-18 ELISA kit (Category No. PI558), a human IL-10 ELISA kit (Category No. PI528), and human IL-33 ELISA kit (Category No. PI631), according to the manufacturer's instructions (Beyotime Biotechnology, China).

\section{Real-time polymerase chain reaction (RT-PCR) for determining inflammatory mediators}

Total RNAs were extracted from the M2-type THP-1 cells with the RNeasy Mini RNA Extraction Kit (Category No. 74104; Qiagen, Hilden, Germany), according to the manufacturer's instructions. RNAs were then synthesized to first-strand complementary DNA with QuantiTect Reverse Transcription Kit (Category No. 205311; Qiagen, Germany), according to the manufacturer's instructions. The RT-PCR assay was conducted using the SYBR Green PCR kit (Category No. DRR820A; TaKaRa, Dalian, China) in Applied Biosystems 7500 Fast Real-time PCR system (Foster City, CA, USA). The specific primers for the amplified TNF- $\alpha, N L R P 1, N L R P 3$, caspase-1, ASC, and $\beta$-actin are listed in Table 1. The transcriptions of targeted genes were normalized to $\beta$-actin, and the relative values were calculated with the previously described $2^{-\Delta \Delta C t}$ (Livak) method (16).

\section{Plasmid construction and lentivirus packaging}

The lentiviral vector PDS019-pL-shRNA-F (NovoBio Scientific, Shanghai, China) was applied to synthesize APE1 shRNA-containing plasmid (PDS019_pL_shRNA_GFPhAPE1), as described in a previously published study (17). We used 3 pairs of primers based on the APE1 gene sequence (NM_080649.2), and synthesized 3 lentiviral plasmids, including VL1275-PDS019_pL_shRNA_GFP-hAPE1-446, VL1276-PDS019_pL_shRNA_GFP-hAPE1-609, and VL1277-PDS019_pL_shRNA_GFP-hAPE1-839 (APE1 ${ }^{\text {shRNA }}$ ) (Figure 1A). By examining interference efficiency, we found that APE $1^{\text {shRNA }}$ demonstrated the highest interference efficiency $(81.82 \%)$ (Figure 1B), as seen in the following experiments. Briefly, 1 day before the transfection of APE1 $1^{\text {shNA }}$, 293T cells (Shanghai Institute of Cells, Chinese Academy of Science, Shanghai, China) were seeded onto a 10-cm dish (Corning-Costar, Corning, NY, USA). The APE $1^{\text {shRNA }}$ plasmid and packing plasmids were transfected into $293 \mathrm{~T}$ cells, according to the manufacturer's instructions. Appropriately $72 \mathrm{~h}$ post-transfection, the supernatants of $293 \mathrm{~T}$ cells were harvested by centrifuging at 2,000 $\times \mathrm{g}$ for $5 \mathrm{~min}$ at $4{ }^{\circ} \mathrm{C}$. Cell supernatants were purified with a $0.5-\mu \mathrm{m}$ syringe filter, and products were re-centrifuged at $20,000 \times \mathrm{g}$ at $4^{\circ} \mathrm{C}$ for $1 \mathrm{~h}$. The APE $1^{\text {shRNA }}$ were packaged into lentiviral.

\section{Activation of inflammasomes}

In the present study, the M2-type THP-1 cells were seeded 
A

\begin{tabular}{cl}
\hline Primers & \multicolumn{1}{c}{$\begin{array}{c}\text { Sequences (5‘-3’) } \\
\text { hAPE1-446-F(NP9172) }\end{array}$} \\
hAPE1-446-R(NP9173) & AAAAGCAAACCTGCCACACTCAAGATTCGTCTTGAGTGTGGCAGGTTTGC \\
hAPE1-609-F(NP9174) & CACCGCCTGGACTCTCTCATCAATACGAATATTGATGAGAGAGTCCAGGC \\
hAPE1-609-R(NP9175) & AAAAGCCTGGACTCTCTCATCAATATTCGTATTGATGAGAGAGTCCAGGC \\
hAPE1-839-F(NP9176) & CACCGGGATGAAGCCTTTCGCAAGTCGAAACTTGCGAAAGGCTTCATCCC \\
hAPE1-839-R(NP9177) & AAAAGGGATGAAGCCTTTCGCAAGTTTCGACTTGCGAAAGGCTTCATCCC \\
\hline
\end{tabular}

B

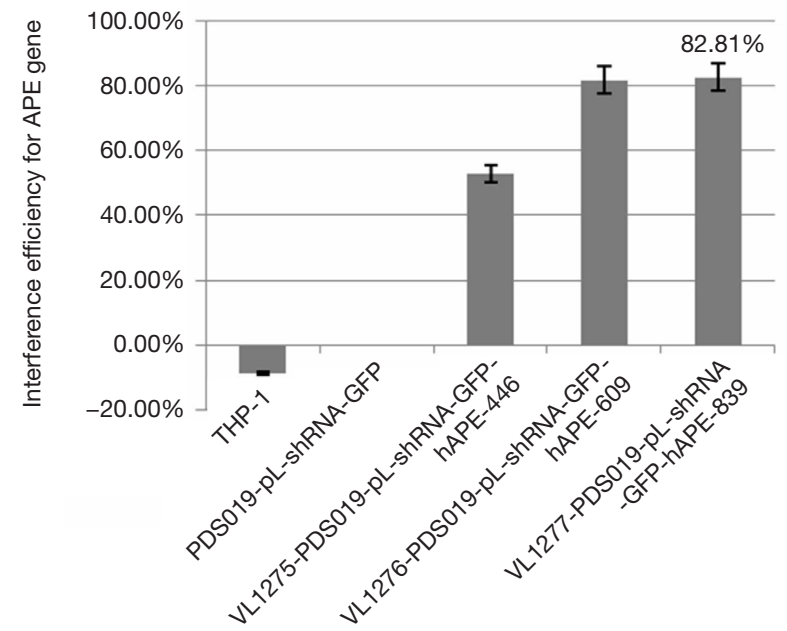

Figure 1 Primers for plasmid construction and evaluation for interference efficiency. (A) Three pairs of primers. (B) Examination for the interference efficiency of 3 interference plasmids.

at a density of $1 \times 10^{6}$ cells $/ \mathrm{mL}$ medium and cultured on 6-well plates. M2-type THP-1 cells were transfected with lentiviral vector APE $1^{\text {shRNA }}$ for $24 \mathrm{~h}$ and then incubated with lipopolysaccharide (LPS) $(100 \mathrm{ng} / \mathrm{mL}) / \mathrm{APE} 1$ inhibitor (E3330, $20 \mu \mathrm{M}$ ) for another $24 \mathrm{~h}$. About $2 \mathrm{~h}$ post-priming, a total of $5 \mathrm{mM}$ ATP was used to incubate the THP-1 cells for $45 \mathrm{~min}$, and the conditioned medium of each well was collected for the following analyses.

\section{Electrophoretic mobility shift assay (EMSA) for AP-1 and

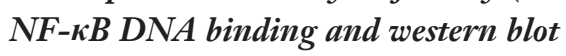

Extraction of the nuclear proteins in M2-type THP1 cells were prepared using NE-PER Nuclear and Cytoplasmic Extraction Reagents, as per the manufacturer's instructions (Category No. 78833, Thermo Scientific Pierce, Rockford, IL, USA) and previously published studies $(18,19)$. Briefly, AP-1 and NF-кB DNA binding in M2-type THP-1 cells was evaluated using EMSA with associated probes. The biotin-labeled AP-1 probes were synthesized and are follows: wild AP-1 probe-sense: 5'-CATTTGGAAAATCCAGCTATG-3', anti-sense: 5'-CATAGCTGGATTTTCCAAATG-3'; cold AP-1 probesense: 5'-CATTTGGAAAATCCAGCTATG-3', anti-sense: 5'-CATAGCTGGATTTTCCAAATG-3'; and mutant AP-1 probe-sense: 5'-CATTTTCAAAATAGAGCTATG-3', anti-sense: 5'-CATAGCTCTATTTTGAAAATG-3'. Specificity for the AP-1 and NF- $\mathrm{KB}$ binding was examined using the competition assay. To evaluate the subunit composition for the DNA-binding molecules, a specific antibody, anti-human NF- $\mathrm{KB}$ p105/p50 antibody (Category No. ab32360, RRID:AB_776748, Abcam Biotechnology, Cambridge, MA, USA) was employed for the super shift assay. The above samples were loaded on non-denaturating $6 \%$ polyacrylamide gels and let free probe running till reaching the end of gel. The AP-1 and NF- $\mathrm{BB}$ bands 
were examined using the LightShift Chemiluminescent EMSA Kit, (Category No. 20148; Thermo Fisher Scientific, USA), while the bands were quantified using densitometry with Quantity-One 1-D software (version: 4.6.9; Bio-Rad, Hercules, CA, USA). For western blot, Commercial antibodies were purchased from indicated vendors: anti-NLRP1 antibody (Category No. ab16091, RRID:AB_302253, Abcam Biotechnology, Cambridge, MA, USA); anti-NLRP3 antibody (Category No. ab214185, RRID:AB_2819003, Abcam Biotechnology, Cambridge, MA, USA); anti-pro caspase-1 + p10 antibody (Category No. ab179515, RRID:AB_2884954, Abcam Biotechnology, Cambridge, MA, USA); GAPDH (Category No. 2118, RRID:AB_561053, Cell Signaling Technology, Danvers, MA, USA); anti-APE1 antibody (Category No. ab194, RRID:AB_302694, Abcam Biotechnology, Cambridge, MA, USA); anti-NF- $\mathrm{KB}$ p65 antibody (Category No. ab16502, RRID:AB_443394, Abcam Biotechnology, Cambridge, MA, USA); anti-c-Jun antibody (Category No. ab32137, RRID:AB_731608,Abcam Biotechnology, Cambridge, MA, USA)

\section{Dual-luciferase reporter assay}

The 3' untranslated region (UTR) of the APE1 gene was cloned into firefly luciferase reporter vector to generate the recombinant vector of PDS160_pL6.3-CMV-EGFPT2A-Rluc, which was assigned as the wide-type vector. $\mathrm{APE} 1^{\text {shRNA }}$ was assigned as the mutant-type vector, and used for dual-luciferase reporter assay. The $293 \mathrm{~T}$ cells were cultured for $24 \mathrm{~h}$ on 24-well plates and co-transfected using the PDS160_pL6.3-CMV-EGFP-T2A-Rluc (or APE1 ${ }^{\text {shRA }}$ ) vector and VL1471_PDS074_pL-TO-IRES-Luc- NLRP3 (or PDS019 vector as control) mimics. Transfections were conducted with the Lipofectamine 2000 reagent (Invitrogen, Carlsbad, CA, USA). At 48 h post-transfection, the dualluciferase reporter assay system (Promega, Madison, MI, USA) and the Turner Designs TD-20/20 Luminometer (Promega, USA) were used to verify luciferase activity normalizing to the Renilla.

\section{Statistical analysis}

In the present study, each experimental condition was independently repeated three times, data derived from experiments or tests were defined as mean \pm standard deviation and analyzed with SPSS software version 20.0 (SPSS, Chicago, IL, USA). Data were analyzed with the
Bonferroni post-hoc validated analysis of variance test to compare differences between groups. $\mathrm{P}<0.05$ was considered statistically significant.

\section{Results}

\section{APE1 induces LPS-induced pro-inflammatory cytokine production}

LPS treatment for THP-1 cells induced significantly increased levels of pro-inflammatory cytokines, including TNF- $\alpha$ (Figure 2A), IL1- $\beta$ (Figure 2B), IL-18 (Figure 2C), IL-10 (Figure 2D), and IL-33 (Figure 2E), compared with those of THP-1 cells without LPS treatments (all $\mathrm{P}<0.05$ ). We employed 2 reagents (APE1 inhibitor E3330 and APE $1^{\text {shRNA }}$ ) for blocking APE1 expression in the THP-1 cells. The results verified that both E3330 (at dosage of 2 and $10 \mu \mathrm{M}$ ) and APE1 $1^{\text {shRNA }}$ induced the LPStriggered production of pro-inflammatory cytokine TNF- $\alpha$ (Figure $2 A$ ), IL-1 $\beta$ (Figure $2 B$ ), and IL-18 (Figure 2C) (all $\mathrm{P}<0.05)$. However, there were no significant effects of E3330 and APE $1^{\text {shRNA }}$ treatment on the production of IL-10 (Figure 2D) and IL-33 (Figure 2E) (all $\mathrm{P}>0.05$ ).

\section{APE1 promotes LPS-induced NLRP3 inflammasome activation by modulating gene transcription of NLRP3- associated molecules}

According to the RT-PCR findings, the transcriptions of inflammatory factors, including $T N F-\alpha, N L R P 1$, and $N L R P 3$, were obviously increased compared with those of THP-1 cells without LPS treatments (all $\mathrm{P}<0.05$ ) (Figure $3 A$ ). TNF- $\alpha$ gene transcriptions were remarkably suppressed in both THP-1 cells undergoing E3330 and APE $1{ }^{\text {shRNA }}$ treatment compared with those in THP-1 cells without treatments $(\mathrm{P}<0.05)$ (Figure $3 A)$. Meanwhile, both E3330 and APE1 ${ }^{\text {shRNA }}$ treatments also significantly inhibited NLRP3 gene transcriptions in THP-1 cells compared with those in THP-1 cells without the above treatments $(\mathrm{P}<0.05)$ (Figure $3 A)$. However, there were no obvious effects of E3330 or APE1 ${ }^{\text {shRNA }}$ treatment on the gene transcription of the NLRP1 molecule in THP-1 cells (Figure 3A).

Moreover, the NLRP3-associated molecules, caspase-1 and $A S C$, were also determined in LPS-treated THP-1 cells. The results showed that both E3330 (in a dose-dependent manner) and APE1 $1^{\text {shRNA }}$ could decreased caspase-1 (Figure 3B) and $A S C$ (Figure 3C) gene transcription compared with 
A

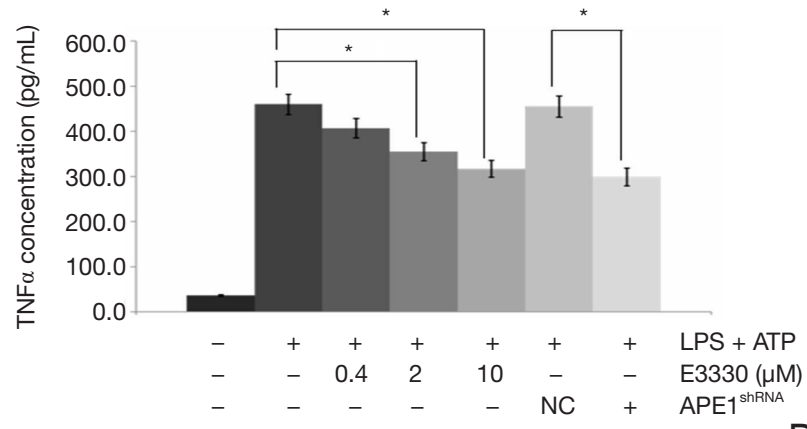

C

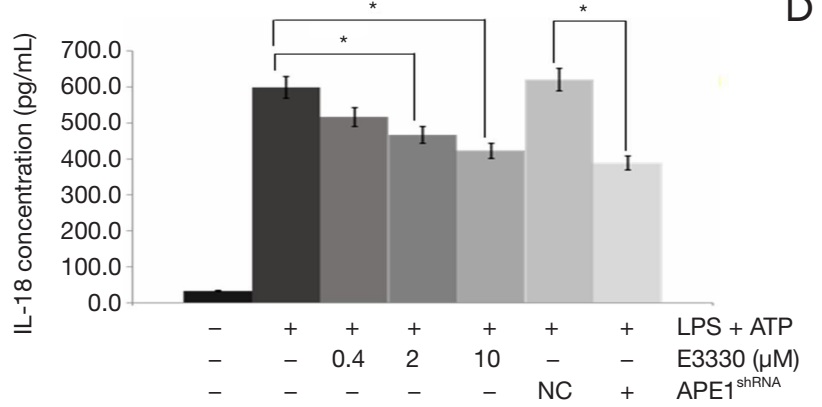

$\mathrm{E}$

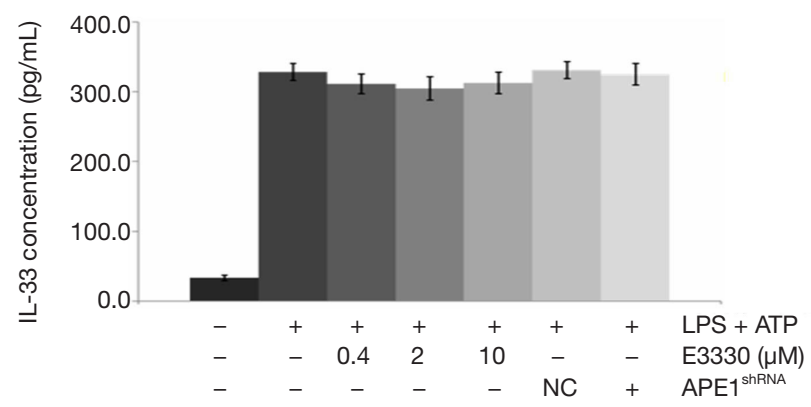

B
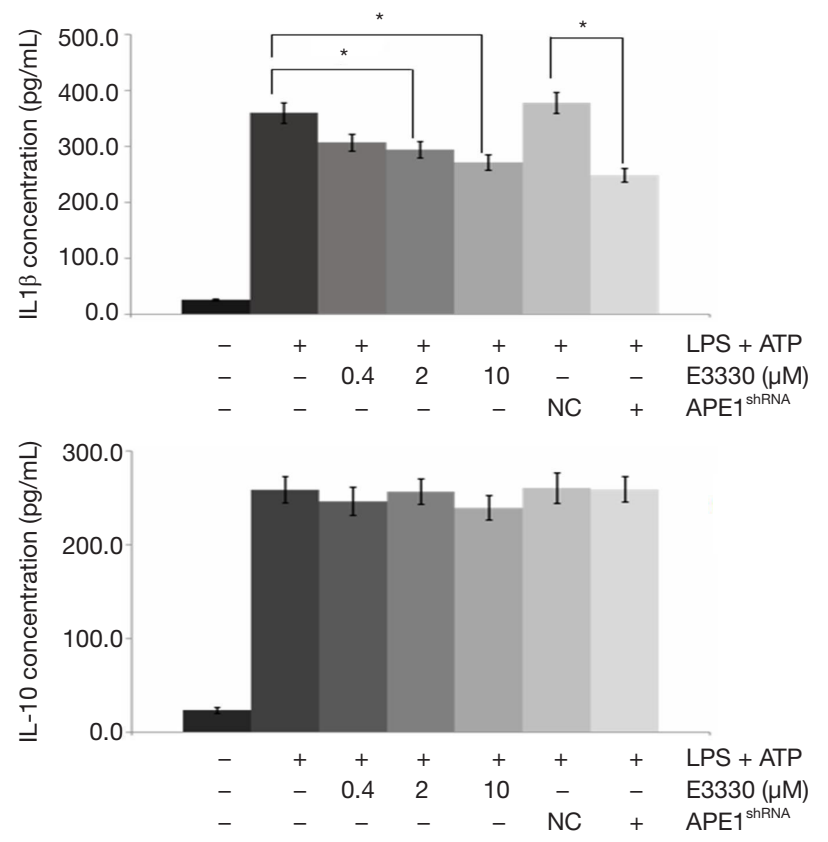

Figure 2 Apurinic/apyrimidinic endonuclease 1/reduction-oxidation effector factor-1 triggered lipopolysaccharide-induced proinflammatory cytokine production in THP-1 cells. Pro-inflammatory cytokine production was detected using enzyme-linked immunosorbent assay (ELISA). (A) Production and statistical analysis of tumor necrosis factor- $\alpha$, interleukin (IL)-1 $\beta$ (B), IL-18 (C), IL-10 (D), and IL-33 (E). ${ }^{*} \mathrm{P}<0.05$ represents significant differences between the two groups.

those in LPS-treated THP-1 cells.

\section{APE1 enhances LPS-induced NLRP3 inflammasome activation by regulating NLRP3 and caspase-1 protein expression}

In the present study, the protein expressions of NLRP1, NLRP3, and caspase-1 were also detected using western blot assay (Figure 4A). The findings indicated that both E3330 (in a dose-dependent manner) and APE $1^{\text {shRNA }}$ could remarkably increase the NLRP3 protein expression (Figure $4 B$ ) and caspase-1 protein expression (Figure 4C) compared with those in the LPS-treated THP-1 cells $(\mathrm{P}<0.05)$. However, no obvious effects of E3330 and APE1 $1^{\text {shRNA }}$ on NLRP1 protein expression were observed in the LPS-treated THP1 cells $(\mathrm{P}>0.05)$ (Figure 4B).

\section{APE1 improves NLRP3 activity by modulating the interaction between NLRP3 and NF- $\mathrm{BB}$}

To verify whether NLRP3 activation was mediated by the activation of NF- $\kappa \mathrm{B}$, the effects of anti-NF- $\kappa \mathrm{B}$ antibody on NLRP3 activation were determined using the EMSA method. The EMSA results showed that NLRP3 


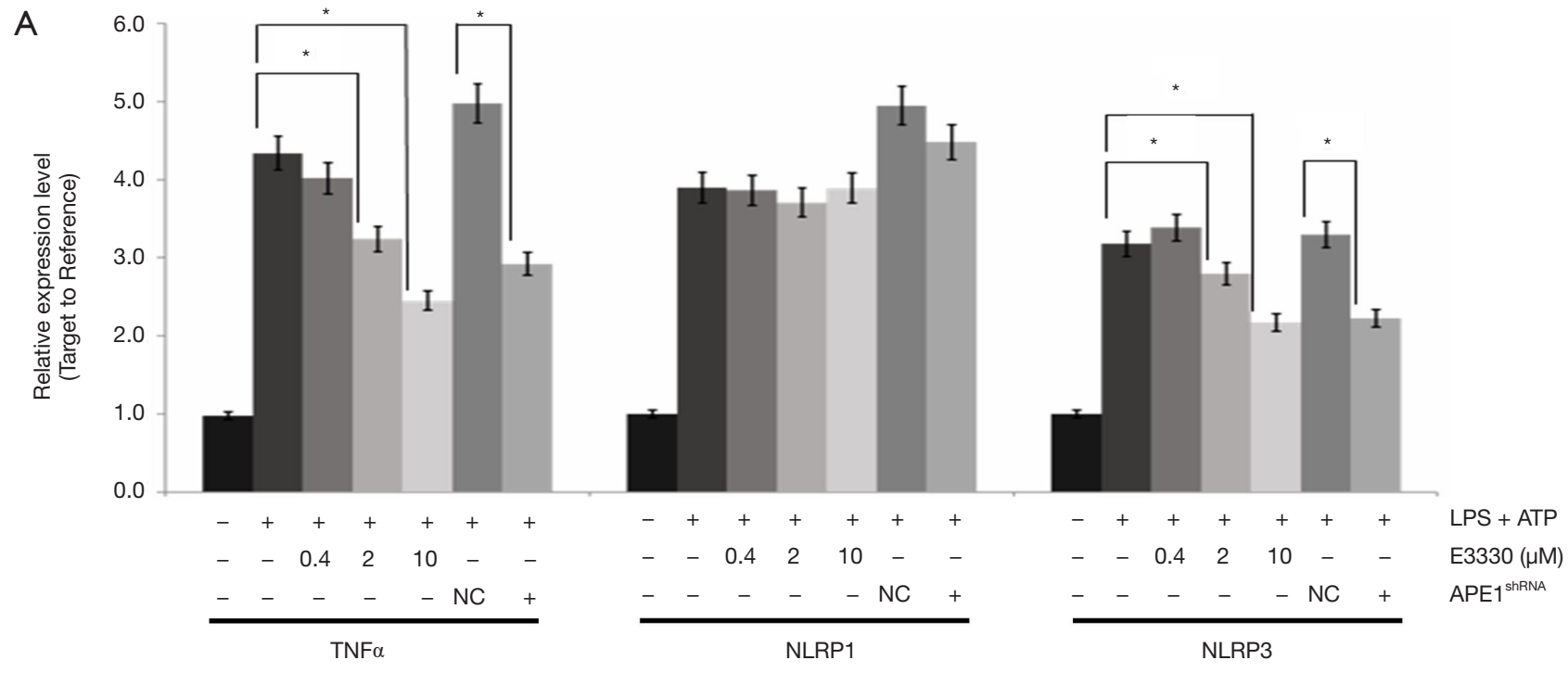

B

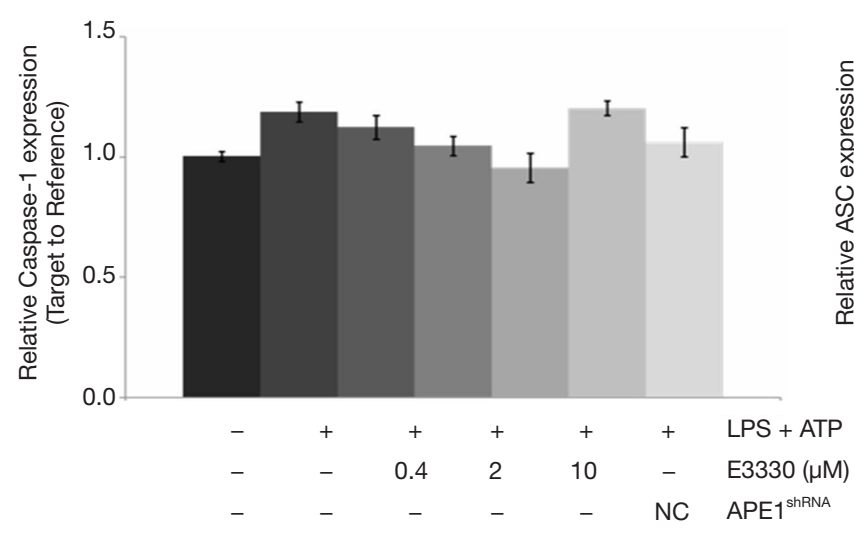

C

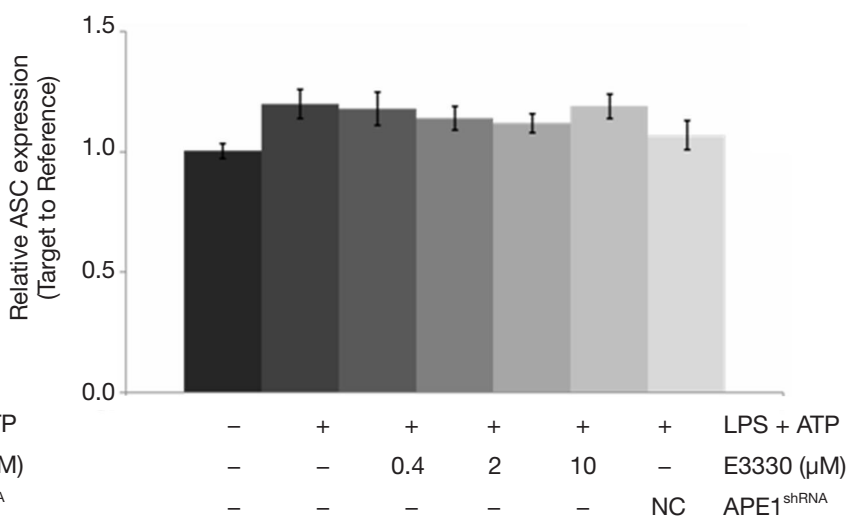

Figure 3 Effects of apurinic/apyrimidinic endonuclease 1/reduction-oxidation effector factor-1 (APE1) on the gene transcriptions of NLR family pyrin domain containing 3 (NLRP3)-associated molecules. APE1 inhibitor (E3330) and plasmid silencing APE1 gene (APE1 ${ }^{\text {shRNA }}$ ) were used to treat THP-1 cells. Gene transcriptions of NLRP3-associated molecules, including tumor necrosis factor- $\alpha$ (TNF- $\alpha$ ), NLR family pyrin domain containing 1 (NLRP1), NLRP3, caspase-1, and apoptosis-associated speck-like protein containing a card (ASC) were determined using reverse transcription-polymerase chain reaction assay. (A) Statistical analysis of the gene transcriptions of TNF- $\alpha$, NLRP1, and NLRP3. (B) Statistical analysis of the gene transcriptions of caspase-1. (C) Statistical analysis of the gene transcriptions of ASC. * $\mathrm{P}<0.05$ represents significant differences between the two groups.

could interact with NF- $\mathrm{BB}$ (Figure $5 A$ ). The chromatin immunoprecipitation (ChIP) findings showed that APE1 improved NLRP3 activity by triggering the interaction between NLRP3 and the NF- $\mathrm{KB}$ molecule (Figure $5 B$ ). Moreover, the dual-luciferase reporter assay also indicated that both E3330- and APE1 $1^{\text {shRNA }}$-treated THP-1 cells demonstrated an interaction between APE1 and the NLRP3 molecule (Figure 5C). Therefore, on the basis of the results, we found that APE1 could improve NLRP3 activity by its redox regulation of $\mathrm{NF}-\kappa \mathrm{B}$.

\section{APE1 upregulates NLRP3 activity through its redox modulation of $N F-\kappa B$}

APE1-associated transcriptional factors, including p65 and NF- $\mathrm{BB}$, were administrated to the THP-1 cells, and expressions of NLRP3, p65, and c-Jun were determined using western blot assay (Figure 6A). The statistical analysis results showed that AP-1 inhibitor treatment decreased NLRP3 expression in a dose-dependent manner; however, without significant changes $(\mathrm{P}>0.05)$ (Figure 6B). The 


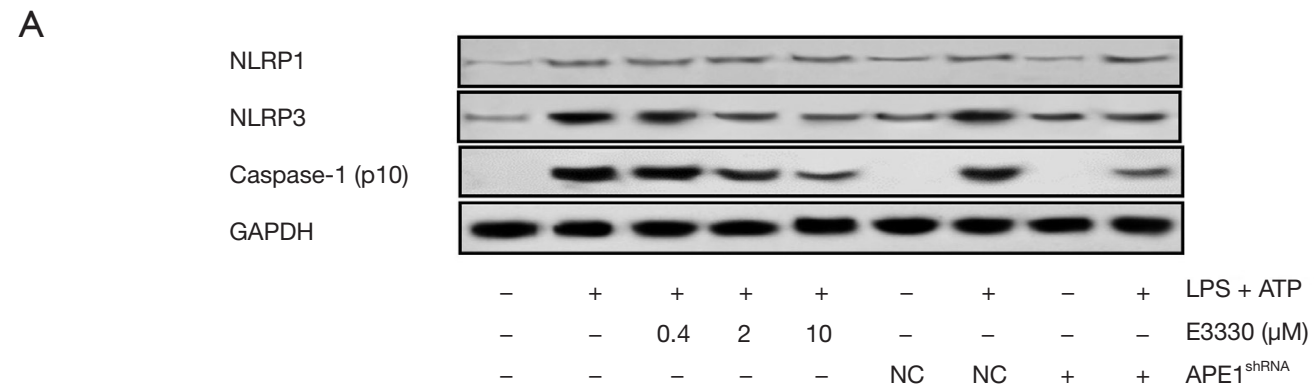

B

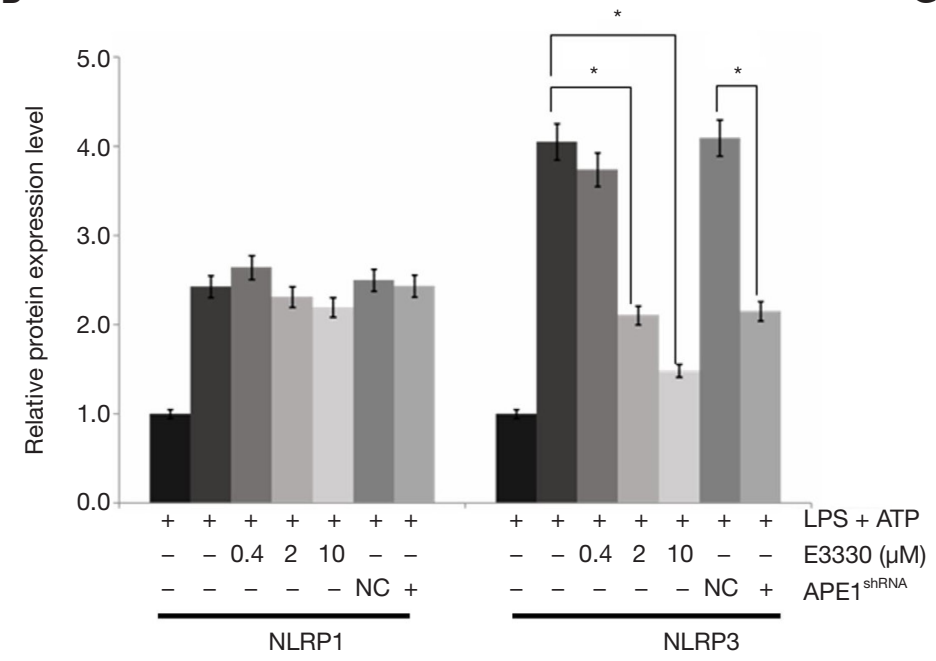

C

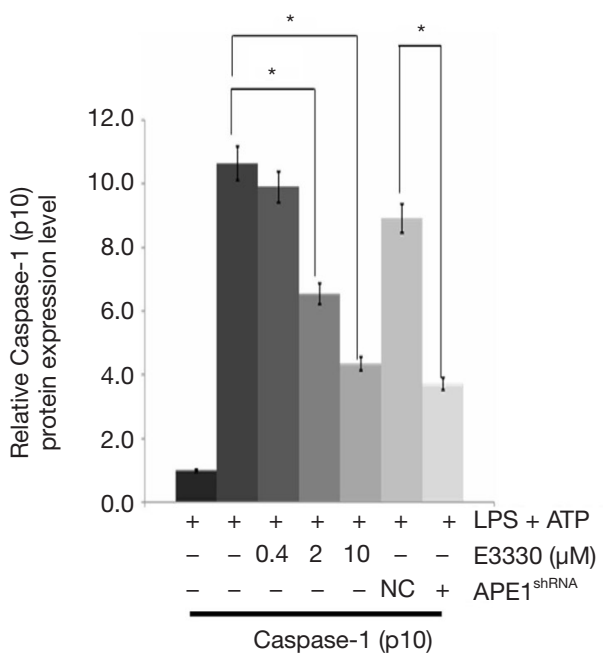

Figure 4 Promoted effects of apurinic/apyrimidinic endonuclease 1/reduction-oxidation effector factor-1 on lipopolysaccharide (LPS)induced NLR family pyrin domain containing 3 (NLRP3) inflammasome in LPS-treated THP-1 cells. Protein expressions of NLRP3 and caspase-1 were examined with western blot assay (A). (A) Statistical analysis of NLR family pyrin domain containing 1 and NLRP3 protein expression in THP-1 cells. (C) Statistical analysis of caspase- 1 protein expression in THP-1 cells. ${ }^{*} \mathrm{P}<0.05$ represents significant differences between the two groups.

administration of the NF- $\kappa \mathrm{B}$ inhibitor significantly reduced NLRP3 expression compared with that in LPS-treated THP1 cells $(\mathrm{P}<0.05)$ in a dose-dependent manner (Figure 6B).

\section{APE1 promotes LPS-induced NLRP3 inflammasome activation through an $\mathrm{NF}-{ }^{\mathrm{K}} \mathrm{B}$-dependent pathway}

To confirm APE1-triggered NLRP3 inflammasome activation, NLRP3 gene transcription was examined in THP-1 cells undergoing APE1 treatment and/or NF- $\mathrm{KB}$ siRNA interference treatment using RT-PCR assay. The results indicated that APE1 treatment could obviously increase NLRP3 gene transcription in LPS-treated THP-1 cells compared with that in cells without APE1 administration (Figure 7A). However, the increased effect of APE1 treatment on NLRP3 gene transcription was obviously blocked by the additional treatment of NF$\kappa \mathrm{B}$ siRNA interference (Figure $7 A$ ). The RT-PCR results also showed that NF- $\kappa B$ siRNA interference treatment significantly inhibited APE1 administration and triggered a stronger effect on NLRP3 gene transcription $(\mathrm{P}<0.05)$ (Figure $7 B$ ). Moreover, we also found that APE1 promoted LPS-induced NLRP3 inflammasome activation by increasing the secretion of NF- $\mathrm{\kappa B}$-mediated inflammatory factors IL-1 $\beta$ and IL-18 (Figure 7C).

\section{Discussion}

In the tumor microenvironment, TAMs communicating with tumors commonly affect the development and 


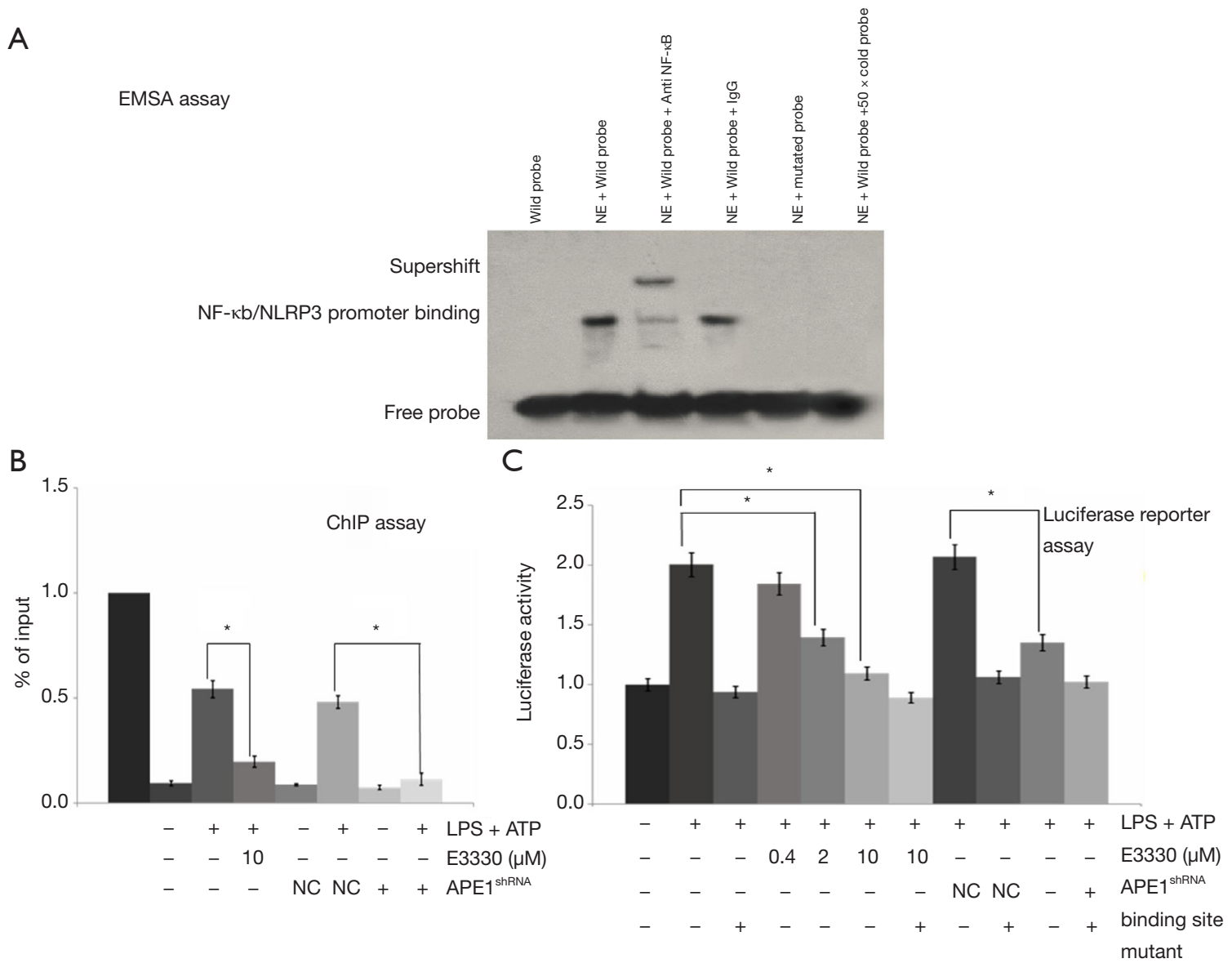

Figure 5 Determination for the effects of apurinic/apyrimidinic endonuclease $1 /$ reduction-oxidation effector factor-1 on the interaction between NLR family pyrin domain containing 3 (NLRP3) and nuclear factor- $\kappa \mathrm{B}(\mathrm{NF}-\kappa \mathrm{B})$ in lipopolysaccharide-treated THP-1 cells. (A) Interaction between NLRP3 and NF- $\mathrm{B}$, as determined by electrophoretic mobility shift assay. (B) Interaction between NLRP3 and NF- $\mathrm{B}$, as determined by chromatin immunoprecipitation. (C) Interaction between NLRP3 and NF- $\mathrm{B}$, as determined with dual-luciferase reporter assay. ${ }^{*} \mathrm{P}<0.05$ represents significant differences between the two groups.

progression of cancers (20,21). Therefore, the polarization of macrophages is critical for therapeutic effects of reagents in cancer treatment. In the present study, THP-1 cells were polarized into the M2 subset macrophages, which were then incubated with APE1 siRNA interference or APE1 inhibitor. The DNA repair system is crucial for genomic stabilization and genomic integrity; however, the high levels of DNA repair might cause drug resistance or radiation resistance in cancer cells (22). Therefore, reduction in DNA repair capability can indirectly decrease the therapeutic effects of anti-tumor drugs. According to previous reports, DNA repair-associated molecules are effective targets for cancer treatment $(23,24)$. Of these molecules, APE1 is considered to be a key target because of its role in the DNA-base excision repair process, and could exert oxidation-reduction modifying activity on a series of transcriptional factors $(25,26)$. APE1 can also activate AP$1, \mathrm{NF}-\kappa \mathrm{B}$, and other transcription factors through redox, therefore regulating cell proliferation and differentiation, as well as inflammatory response. Therefore, we would like to evaluate the effects of APE1 on the inflammasome in THP1 cells and explore the associated mechanisms.

In the present study, we first treated the THP-1 cells with LPS to generate the inflammatory status, and then incubated cells with APE-1 inhibitor (E3330) and/or APE$1^{\text {shRNA }}$ (27). According to the ELISA results, both E3330 


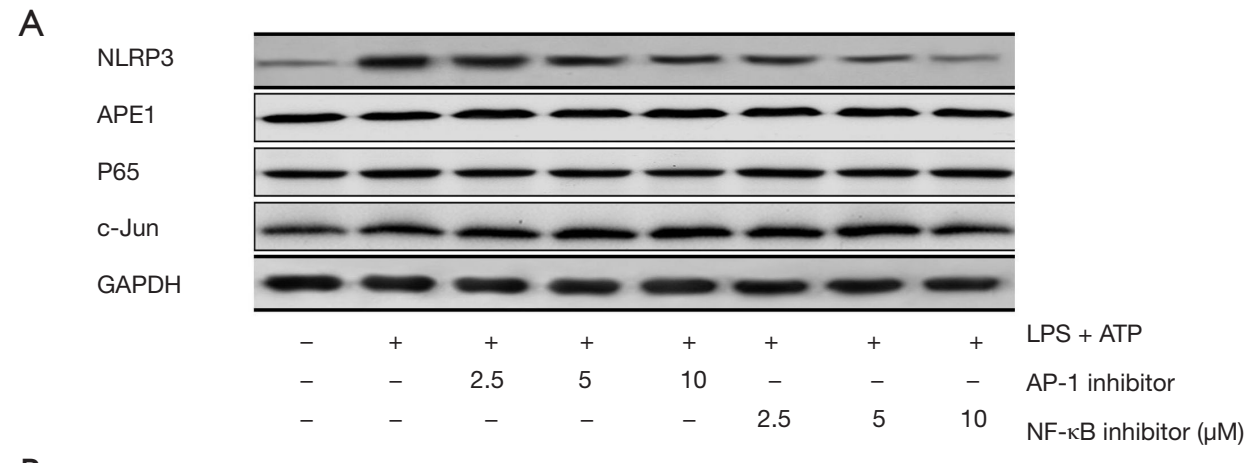

B

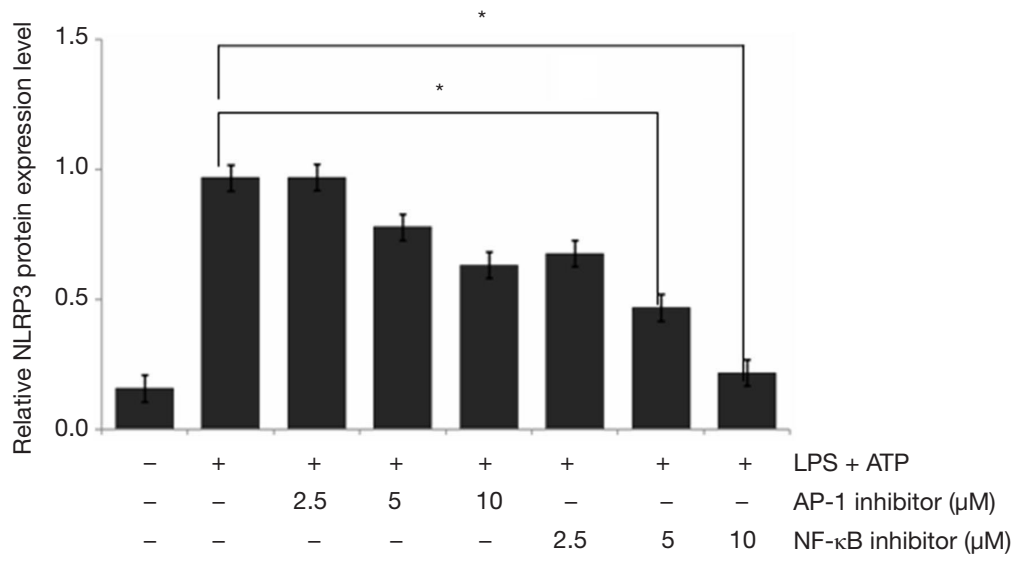

Figure 6 Apurinic/apyrimidinic endonuclease 1/reduction-oxidation effector factor-1 regulated NLR family pyrin domain containing 3 (NLRP3) activity by modulating the nuclear factor- $\kappa$ B molecule. (A) Western blot assay for the expression of NLRP3, p65, and c-Jun in the lipopolysaccharide-treated THP-1 cells. (B) Statistical analysis of NLRP3 expression. ${ }^{*} \mathrm{P}<0.05$ represents significant differences between the two groups.

and APE $1^{\text {shRNA }}$ remarkably induced the LPS-triggered production of pro-inflammatory cytokines TNF- $\alpha$, IL-1 $\beta$, and IL-18. However, there were no significant effects of E3330 and APE1 ${ }^{\text {shRNA }}$ treatment on the production of IL-10 and IL-33. These findings suggest that APE1 induced LPSinduced pro-inflammatory cytokine production. However, Sawakami et al. reported that APE-1 silencing causes an obvious increase in the expression of TNF- $\alpha$ and IL- $1 \beta$ in L-02 cells, which is contrast to our results (28). The difference in results could be a result of the different applied cell lines, which need further clarification in future studies.

Based on the APE-1 induced production of proinflammatory cytokine and potential binding site with the NLRP3 gene, we speculated that APE-1 might also be involved in the activation of NLRP3 inflammasome (29). Both E3330 and APE1 ${ }^{\text {shRNA }}$ treatments also significantly inhibited the $T N F-\alpha$ gene and $N L R P 3$ gene transcriptions in THP-1 cells compared with those in THP-1 cells without the above treatments; however, without effects on NLRP1 gene transcription. Moreover, the NLRP3 inflammasomeassociated molecules, caspase-1 and ASC (30), were also determined in LPS-treated THP-1 cells. The results showed that both $\mathrm{E} 3330$ and $\mathrm{APE} 1^{\text {shRNA }}$ could decreased caspase-1 and $A S C$ gene transcription. These results suggest that APE1 could promote LPS-induced NLRP3 inflammasome activation by modulating the gene transcription of NLRP3-associated molecules.

A bioinformatics analysis showed that the NLRP3 gene promoter contains potential AP-1 and NF- $\mathrm{\kappa B}$ binding sites (14), therefore, the effect of APE1 on NLRP3 inflammasome activation might be mediated by the binding of NLRP3 with transcription factors. The EMSA findings, 
A

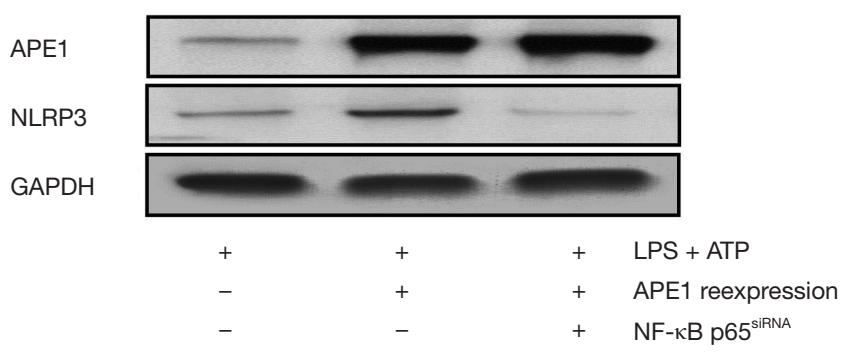

B

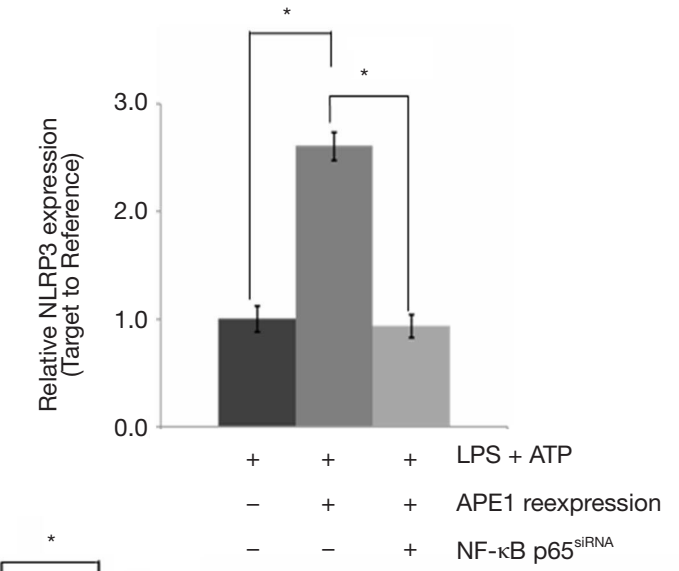

C

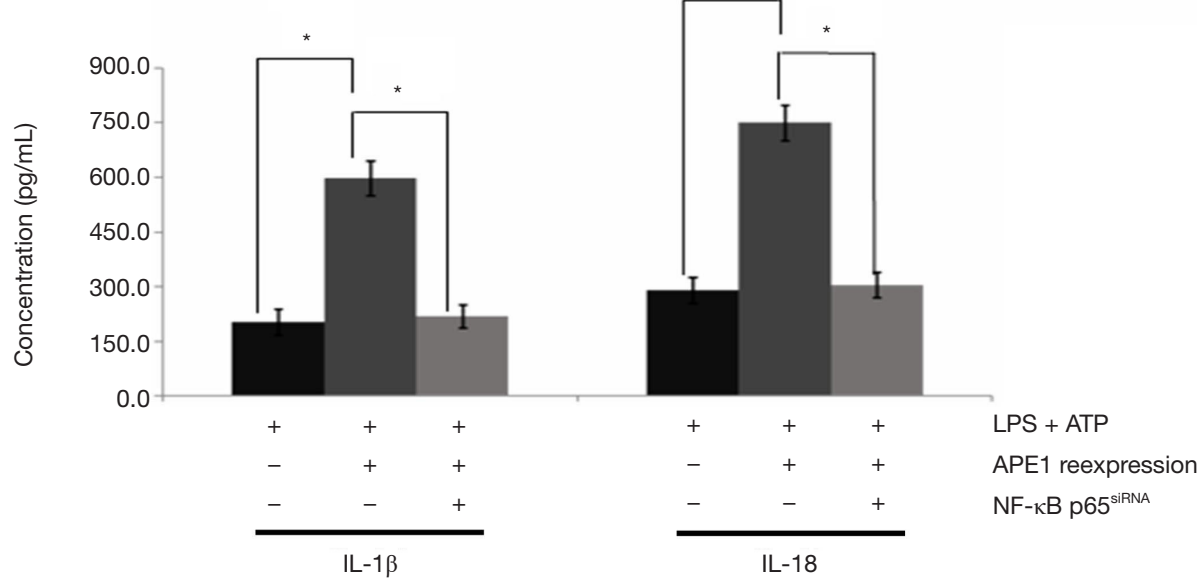

Figure 7 Apurinic/apyrimidinic endonuclease 1/reduction-oxidation effector factor-1 (APE1) promoted the lipopolysaccharide (LPS)induced NLR family pyrin domain containing 3 (NLRP3) inflammasome activation by triggering the nuclear factor- $\kappa \mathrm{B}-\mathrm{dependent} \mathrm{signaling}$ pathway. (A) Western blot assay for APE1 and NLRP3 expression in LPS-treated THP-1 cells. (B) Statistical analysis of NLRP3 expression. (C) Determination of section of inflammatory factors interleukin (IL)- $1 \beta$ and IL-18 using enzyme-linked immunosorbent assay. * $\mathrm{P}<0.05$ represents significant differences between the two groups.

ChIP results, and dual-luciferase reporter assay found that APE-1 could improve NLRP3 activity by triggering the interaction between NLRP3 and the NF- $\mathrm{NB}$ molecule. Therefore, APE1 could improve NLRP3 activity by its redox regulation of NF- $\mathrm{\kappa B}$. Moreover, we also found that APE1 upregulated NLRP3 activity through its redox modulation of NF- $\kappa \mathrm{B}$, which also confirmed the interaction of NLRP3 and NF- $\kappa$ B.

To evaluate whether the NF- $\kappa \mathrm{B}$ signaling pathway participates in APE1-triggered NLRP3 inflammasome activation (31), NLRP3 gene transcription was examined in THP-1 cells undergoing APE1 treatment and/or NF- $\mathrm{\kappa B}$ siRNA interference treatment. APE1 treatment obviously increased NLRP3 gene transcription in LPS-treated THP1 cells; however, this increased effect was obviously blocked by the additional treatment of NF- $\kappa \mathrm{B}$ siRNA interference. Moreover, APE1 could also promote LPS-induced NLRP3 inflammasome activation by increasing the section of NF- $\kappa$ B-mediated inflammatory factors IL- $1 \beta$ and IL- 18 . These results suggest that APE1 promotes LPS-induced NLRP3 inflammasome activation through an NF-kBdependent pathway.

\section{Conclusions}

The present study showed that APE1, as an important oxidative stress effector molecule, regulates NLRP3 expression through its redox modification, therefore further promoting the secretion of inflammatory mediators IL- $1 \beta$ and IL-18 via THP-1 cells (Figure 8). The study confirmed 


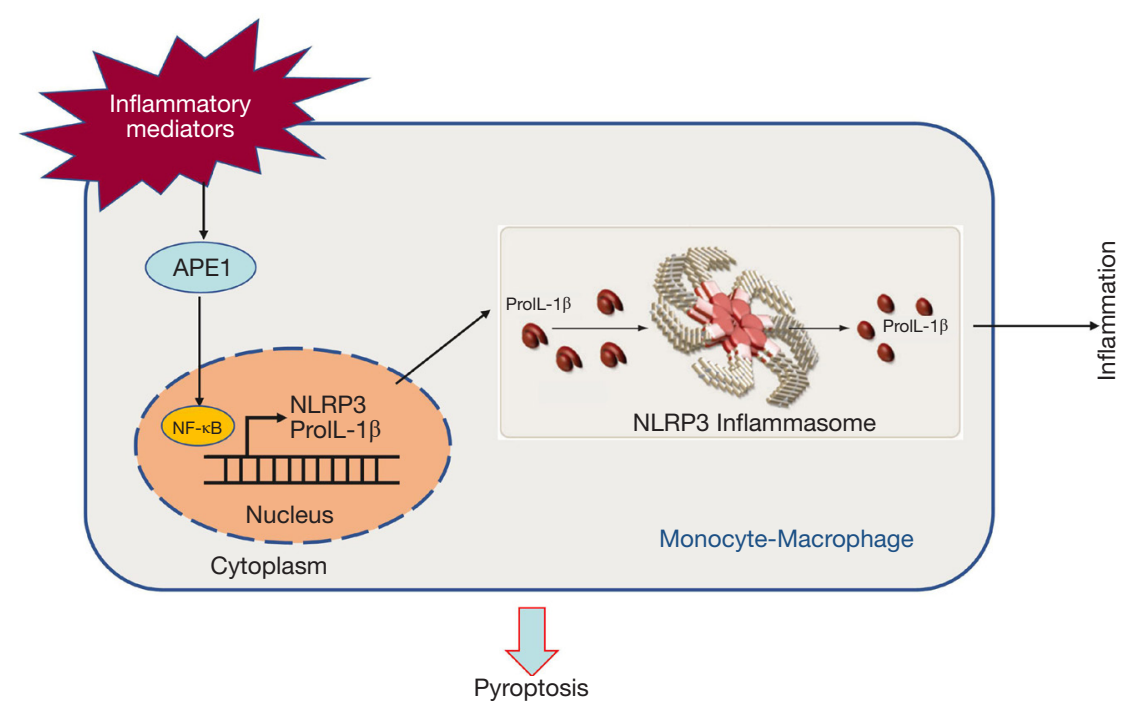

Figure 8 Diagrammatic sketch for the mechanism of apurinic/apyrimidinic endonuclease 1/reduction-oxidation effector factor-1 (APE1) regulating NLR family pyrin domain containing 3 (NLRP3) to promote the secretion of inflammatory mediators by tumor-associated macrophages. Inflammatory mediators stimulate the APE1 molecule, which trigger the interaction between NLRP3 and the nuclear factor- $\kappa \mathrm{B}$ molecule in the cytoplasm of cells. Inflammatory factors, such as pro-inflammatory interleukin-1 $\beta$, were produced, and NLRP3 inflammasome was generated in the macrophages.

that APE1 may regulate the expression of NLRP3 by modulating transcription factor NF- $\mathrm{BB}$. We explored the regulatory mechanism of NLRP3 transcription and secretion of inflammatory mediators in TAMs from a new perspective, and provided theoretical and experimental bases for the design of TAM-targeted therapy, with APE1 as the target molecule.

\section{Acknowledgments}

Funding: The present study was supported by the Youth Program of National Natural Science Foundation of China (Grant No. 81802740), Chongqing Basic Research and Frontier Exploration Project (Chongqing Natural Science Foundation, Grant No. cstc2018jcyjAX0741), Key Technology Project for Prevention and Control of Major Diseases in Chongqing (Grant No. 2019ZX00), and Chongqing Scientific Research Institute Performance Incentive Guidance Project (Grant No. cstc2017jxj1130016).

\section{Footnote}

Reporting Checklist: The authors have completed the MDAR reporting checklist. Available at http://dx.doi.org/10.21037/ atm-20-7752
Data Sharing Statement: Available at http://dx.doi. org/10.21037/atm-20-7752

Conflicts of Interest: All authors have completed the ICMJE uniform disclosure form (available at http://dx.doi. org/10.21037/atm-20-7752). The authors have no conflicts of interest to declare.

Ethical Statement: The authors are accountable for all aspects of the work in ensuring that questions related to the accuracy or integrity of any part of the work are appropriately investigated and resolved. Because the study does not include animals or patients, the ethics committee approval was waived.

Open Access Statement: This is an Open Access article distributed in accordance with the Creative Commons Attribution-NonCommercial-NoDerivs 4.0 International License (CC BY-NC-ND 4.0), which permits the noncommercial replication and distribution of the article with the strict proviso that no changes or edits are made and the original work is properly cited (including links to both the formal publication through the relevant DOI and the license). See: https://creativecommons.org/licenses/by-nc$\mathrm{nd} / 4.0 \%$. 


\section{References}

1. Li G, Liu S, Wang H, et al. Ligustrazine ameliorates lipopolysaccharide-induced neurocognitive impairment by activating autophagy via the PI3K/AKT/mTOR pathway. Int J Mol Med 2020;45:1711-20.

2. Bülbül B, Küçükgüzel İ. Microsomal Prostaglandin E2 Synthase-1 as a New Macromolecular Drug Target in the Prevention of Inflammation and Cancer. Anticancer Agents Med Chem 2019;19:1205-22.

3. Mantovani A, Sozzani S, Locati M, et al. Macrophage polarization: tumor-associated macrophages as a paradigm for polarized M2 mononuclear phagocytes. Trends Immunol 2002;23:549-55.

4. Sawa-Wejksza K, Kandefer-Szerszen M. Tumor-associated macrophages as target for antitumor therapy. Arch Immunol Ther Exp (Warsz) 2018;66:97-111.

5. Pollard JW.Tumour-educated macrophages promote tumour progression and metastasis. Nat Rev Cancer 2004;4:71-8.

6. Kummer JA, Broekhuizen R, Everett H, et al. Inflammasome components NALP 1 and 3 show distinct but separate expression profiles in human tissues suggesting a site-specific role in the inflammatory response. J Histochem Cytochem 2007;55:443-52.

7. Song M, Liu T, Shi C, et al. Bioconjugated Manganese Dioxide Nanoparticles Enhance Chemotherapy Response by Priming Tumor-Associated Macrophages toward M1like Phenotype and Attenuating Tumor Hypoxia. ACS Nano 2016;10:633-47.

8. Roux C, Jafari SM, Shinde R, et al. Reactive oxygen species modulate macrophage immunosuppressive phenotype through the up-regulation of PD-L1. Proc Natl Acad Sci USA 2019;116:4326-35.

9. Schroder K, Tschopp J. The inflammasomes. Cell 2010;140:821-32.

10. Abderrazak A, Syrovets T, Couchie D, et al. NLRP3 inflammasome: from a danger signal sensor to a regulatory node of oxidative stress and inflammatory diseases. Redox Biol 2015;4:296-307.

11. Luo M, Delaplane S, Jiang A, et al. Role of the multifunctional DNA repair and redox signaling protein APE1 in cancer and endothelial cells: small-molecule inhibition of the redox function of Ape1. Antioxid Redox Signal 2008;10:1853-67.

12. O'Hara AM, Bhattacharyya A, Mifflin RC, et al. Interleukin-8 induction by Helicobacter pylori in gastric epithelial cells is dependent on apurinic/ apyrimidinic endonuclease-1/redox factor-1. J Immunol 2006;177:7990-9.

13. Rahman I, Biswas SK, Kirkham PA. Regulation of inflammation and redox signaling by dietary polyphenols. Biochem Pharmacol 2006;72:1439-52.

14. Maharana J, Panda D, De S. Deciphering the ATPbinding mechanism in NLRP-NACHT 3D models using structural bioinformatics approaches. PLoS One 2018; 13:e0209420.

15. Tjiu JW, Chen JS, Shun CT, et al. Tumor-associated macrophage-induced invasion and angiogenesis of human basal cell carcinoma cells by cyclooxygenase-2 induction. J Invest Dermatol 2009;129:1016-25.

16. Livak KJ, Schmittgen TD. Analysis of relative gene expression data using real-time quantitative PCR and the 2(-Delta Delta C(T)) Method. Methods 2001;25:402-8.

17. Cun Y, Zhang Q, Xiong C, et al. Combined use of adenoviral vector Ad35/F35-mediated APE1 siRNA enhances the therapeutic efficacy of adenoviral-mediated p53 gene transfer in hepatoma cells in vitro and in vivo. Oncol Rep 2013;29:2197-204.

18. Deryckere F, Gannon F. A one-hour minipreparation technique for extraction of DNA-binding proteins from animal tissues. Biotechniques 1994;16:405.

19. Videla LA, Tapia G, Rodrigo R, et al. Liver NF-kappa B and AP-1 DNA binding in obese patients. Obesity (Silver Spring) 2009;17:973-9.

20. Almatroodi SA, McDonald CF, Darby IA, et al. Characterization of M1/M2 tumor-associated macrophages (TAMs) and Th1/Th2 cytokine profiles in patients with NSCLC. Cancer Microenviron 2016;9:1-11.

21. Wei X, Yang S, Pu X, et al. Tumor-associated macrophages increase the proportion of cancer stem cells in lymphoma by secreting pleiotrophin. Am J Transl Res 2019;11:6393-402.

22. Cun Y, Dai N, Xiong C, et al. Silencing of APE1 enhances sensitivity of human hepatocellular carcinoma cells to radiotherapy in vitro and in a xenograft model. PLoS One 2013;8:e55313.

23. Belzile JP, Choudhury SA, Cournoyer D, et al. Targeting DNA repair proteins: a promising avenue for cancer gene therapy. Curr Gene Ther 2006;6:111-23.

24. Ding J, Miao ZH, Meng LH, et al. Emerging cancer therapeutic opportunities target DNA-repair systems. Trends Pharmacol Sci 2006;27:338-44.

25. Fishel ML, Kelley MR. The DNA base excision their repair protein Ape1/Ref-1 as a therapeutic and chemopreventive target. Mol Aspects Med 2007;28:375-95.

26. Tell G, Quadrifoglio F, Tiribelli C, et al. The many 


\section{Page 14 of 14}

functions of APE1/Ref-1: not a DNA repair enzyme. Antioxid Redox Signal 2009;11:601-20.

27. Zou GM, Karikari C, Kabe Y, et al. The APE-1/Ref1 redox antagonist E3330 inhibits the growth of tumor endothelium and endothelial progenitor cells: therapeutic implications in tumor angiogenesis. J Cell Physiol 2009;219:209-18.

28. Sawakami T, Sun Z, Inagaki Y, et al. The impact of apurinic-apyrimidinic endonuclease I on hepatocyte immuno-inflammatory factors and cell apoptosis. Biosci Trends 2019;13:539-45.

Cite this article as: Tang Z, Wang Y, Wan Y, Xie Y, Li S, Tao D, Wang C, Wu YZ, Sui JD. Apurinic/apyrimidinic endonuclease $1 /$ reduction-oxidation effector factor-1 (APE1) regulates the expression of NLR family pyrin domain containing 3 (NLRP3) inflammasome through modulating transcription factor NF$\kappa \mathrm{B}$ and promoting the secretion of inflammatory mediators in macrophages. Ann Transl Med 2021;9(2):145. doi: 10.21037/ atm-20-7752

\section{Tang et al. APE1 regulates NLRP3 inflammasome in macrophages}

29. Kelley N, Jeltema D, Duan Y, et al. The NLRP3 inflammasome: an overview of mechanisms of activation and regulation. Int J Mol Sci 2019;20:3328.

30. Sun L, Ma W, Gao W, et al. Propofol directly induces caspase-1 dependent macrophage pyroptosis through the NLRP3-ASC inflammasome. Cell Death Dis 2019;10:542.

31. Afonina IS, Zhong Z, Karin M, et al. Limiting inflammation the negative regulation of NF-kB and the NLRP3 inflammasome. Nat Immunol 2017;18:861-9.

(English Language Editor: R. Scott) 